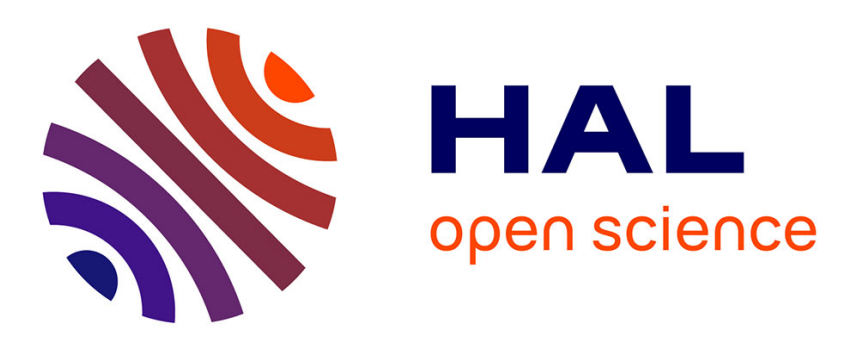

\title{
Thermal Properties of Poly(3-(2'-Ethyl)Hexylthiophene): Study with a Real-Time Combination of Synchrotron X-Ray Scattering and Ultrafast Chip Calorimetry
}

Alexey P Melnikov, Martin Rosenthal, Tim Erdmann, Anton Kiriy, Dimitri

Ivanov

\section{To cite this version:}

Alexey P Melnikov, Martin Rosenthal, Tim Erdmann, Anton Kiriy, Dimitri Ivanov. Thermal Properties of Poly(3-(2'-Ethyl)Hexylthiophene): Study with a Real-Time Combination of Synchrotron X-Ray Scattering and Ultrafast Chip Calorimetry. Key Engineering Materials, 2020, 869, pp.375-381. 10.4028/www.scientific.net/KEM.869.375 . hal-03095881

\section{HAL Id: hal-03095881 https://hal.science/hal-03095881}

Submitted on 7 Jan 2021

HAL is a multi-disciplinary open access archive for the deposit and dissemination of scientific research documents, whether they are published or not. The documents may come from teaching and research institutions in France or abroad, or from public or private research centers.
L'archive ouverte pluridisciplinaire HAL, est destinée au dépôt et à la diffusion de documents scientifiques de niveau recherche, publiés ou non, émanant des établissements d'enseignement et de recherche français ou étrangers, des laboratoires publics ou privés. 


\title{
Thermal properties of poly(3-(2'-ethyl)hexylthiophene): study with a real- time combination of synchrotron X-ray scattering and ultrafast chip calorimetry
}

Alexey P. Melnikov ${ }^{1,2,3, a,{ }^{*}}$, Martin Rosenthal ${ }^{4, b}$, Tim Erdmann ${ }^{5, \mathrm{c}}$, Anton Kiriy ${ }^{5, \mathrm{~d}}$ and Dimitri A. Ivanov $^{1,2,3,6, \mathrm{e}}$

${ }^{1}$ Moscow Institute of Physics and Technology, Institutskiy per. 9, 141700, Dolgoprudny, Russia

${ }^{2}$ Lomonosov Moscow State University, Faculty of Fundamental Physical and Chemical Engineering, GSP-1, 1-51 Leninskie Gory, 119991, Moscow, Russia.

${ }^{3}$ Institute of Problems of Chemical Physics, Russian Academy of Sciences, Semenov Av. 1, 142432, Chernogolovka, Moscow region, Russia

${ }^{4}$ European Synchrotron Radiation Facility (ESRF), 6 rue Jules Horowitz, 38043, Grenoble, France

${ }^{5}$ Institut Makromolekulare Chemie, Leibniz-Institut für Polymerforschung, Hohe Str. 6, 601069, Dresden, Germany

${ }^{6}$ Institut de Sciences des Matériaux de Mulhouse-IS2M, CNRS UMR 7361, Jean Starcky, 15, F68057, Mulhouse, France

르elnikov.al.pe@gmail.com, ${ }^{\text {b }}$ martin.rosenthal@esrf.fr, ${ }^{c}$ erdmann@ipfdd.de, ${ }^{d}$ kiriy@ipfdd.de, eivanov.da@mipt.ru

Keywords: organic semicoductor, X-ray scattering, ultrafast chip calorimetry, structural reorganization

\begin{abstract}
Here we report on reorganization on heating of a perspective organic semiconductor poly(3-(2'ethyl)hexylthiophene) (P3EHT). P3EHT is an analogue of a well-known poly(3-hexylthiophene) (P3HT), which has comparable optoelectronic properties and the advantage of a lower processing temperature. The processes of structural reorganization during heating of P3EHT have been explored with a combination of synchrotron X-ray scattering and ultrafast chip calorimetry. The signature of reorganization has been identified from an increase of d-spacing of 100 peak of the P3EHT unit cell. It was observed that reorganization operates during heating of P3EHT at conventional rates of a DSC experiment (i.e., at $10 \mathrm{deg} / \mathrm{min}$ ), whereas it is largely suppressed at a heating rate of $100 \mathrm{deg} / \mathrm{s}$. Despite the absence of reorganization at high heating rates the calorimetric curves exhibit pronounced double melting, which corroborates the model of the negative pressure building up during crystallization of semi-rigid chain polymers.
\end{abstract}

\section{Introduction}

The family of poly(3-alkylthiophenes) (P3ATs) has attracted significant interest over the last years due to the potential of these polymers for applications in the field of organic photovoltaics and fieldeffect transistors [1]. Among these polymers poly(3-hexylthiophene) (P3HT) has become the classical electron-donor system for several optoelectronic applications [2]. However, despite its 
advantages such as high charge mobility P3HT has a drawback of having relatively high processing temperature related to its melting and glass transitions. Therefore, processing of the active layers of the optoelectronic devices requires annealing at high temperature followed by quenching that can result in generating thermodynamically metastable states. An interesting analogue of P3HT having similar electronic properties is poly(3-(2'-ethyl) hexylthiophene) (P3EHT), which is a polymer possessing the same backbone and ramified ethyl-hexyl side chains [3-4]. The difference in the sidechain architecture of P3EHT brings about a much lower glass transition and melting, which can be beneficial for practical applications. However, the details of the thermal behaviour of P3EHT and in particular its possible structural reorganization during heating should be carefully studied in order to be able to optimize the post-processing protocols of the active layers of photovoltaic devices.

It is noteworthy that P3EHT as well as other P3ATs belong to the family of the semirigid chain semicrystalline polymers which are renowned for their complex thermal behavior. This makes P3EHT similar to the classical representatives of the semirigid chain family such as poly(ethylene terephthalate) (PET), poly(ether ether ketone) (PEEK) and others. These polymers display the socalled double-melting behavior, which has been a highly debated topic in polymer physics since several decades [5-8]. Several models have been put in the past to account for the presence of several endothermic peaks on the DSC heating traces of these polymers. It should be emphasized that the appearance of several melting peaks is not related to the polymorphism and that for many of the semirigid chain polymers only one polymorph has been reported. However, since it was not always known if the structural reorganization takes place during the thermal experiments, the outcome of these studies remain ambiguous. Therefore, one has to completely exclude such reorganization if one seeks for the information relevant to the initial sample state.

Recently, we showed that the details of the thermal behavior in the semirigid chain polymers can be efficiently analyzed if calorimetry is coupled in real time with a structure-sensitive technique such as X-ray scattering [9-10]. Such in operando approach can make it possible addressing the structural nature of each of the thermal events observed in the DSC traces. Also, the access to high heating rates was found to be crucial because the critical heating rates above which the structural reorganization ceases to exist is typically on the order of $1000 \mathrm{~K} / \mathrm{s}$ [10]. The technique capable of reaching such high heating rates is ultra-fast chip calorimetry (FCC) [11]. The technique is based on the use of a MEMStype sensor allowing heating a several-nanogram sample with a system of resistive heaters sputtered on a thin silicon nitride membrane. Previously, we compatibilized the self-made accessory for FCC with a micro-focus synchrotron beamline in order to perform synchronized time-resolved experiments in combination with X-ray scattering [12-13]. In the present work, the developed technique of in-situ FCC with micro-focused X-ray scattering will be employed to analyze the thermal behavior of P3EHT.

\section{Materials and Methods}

P3EHT was synthesized from 3-iodo-5-bromo derivative of the 3-(2'-ethyl)hexylthiophene using isopropyl magnesium chloride catalyst. The reaction was carried out in THF in the temperature range from RT to $40^{\circ} \mathrm{C}$. The resulting molecular weight of P3EHT, as determined by ${ }^{1} \mathrm{H}-\mathrm{NMR}$ is 15.3 $\mathrm{kg} / \mathrm{mol}$. In terms of the polymerization degree this corresponds to $\mathrm{n}=78 \pm 2$. The glass transition temperature of $\mathrm{P} 3 \mathrm{EHT}$ equals $0^{\circ} \mathrm{C}$.

The micro-beam X-ray scattering experiments have been carried out at the ID13 beamline of the European Synchrotron Radiation Facility (ESRF) (Grenoble, France). A schematic representation of our experimental setup is given in Figure 1 (left). The AC and DC FCC experiments can be realized, 
thanks to the home-built electronic interface capable of applying custom temperature programs to the resistive heaters of the nanocalorimetric sensor and performing fast readout of the thermopile signal of the sensor.

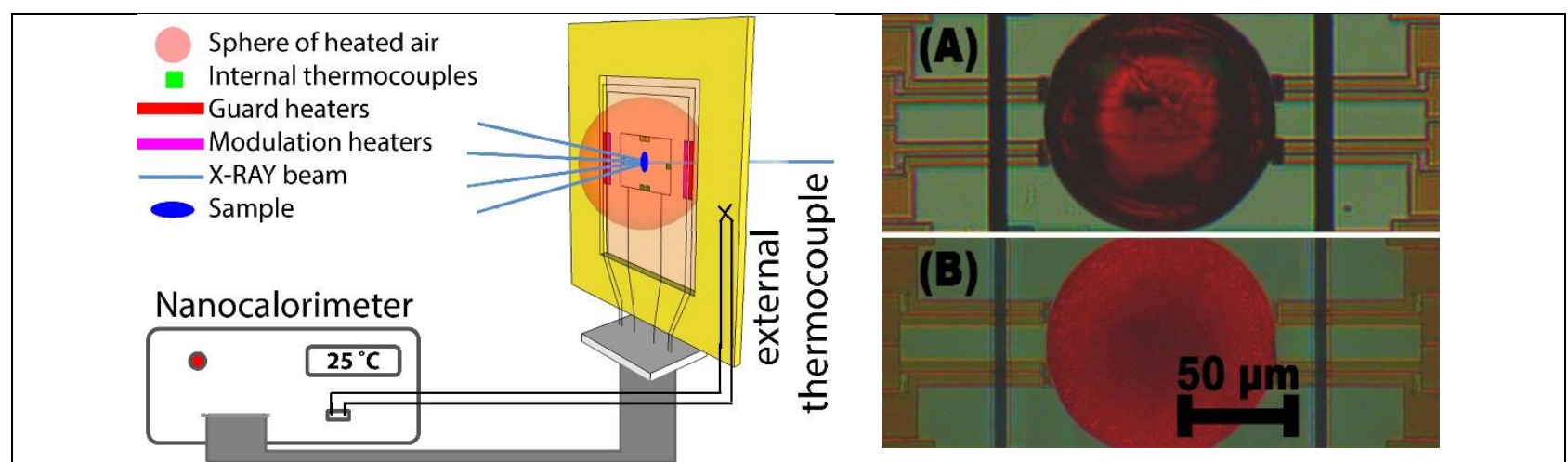

Figure 1. Left: schematics of the experimental setup combining ultrafast calorimetry on a chip (nanocalorimetry) with synchrotron X-ray scattering; Right: example of a P3EHT sample (microdrop) deposited on a MEMS-type sensor for the measurement. The micrographs in A and B stand for the molten state of the sample and the semicrystalline state achieved upon isothermal meltcrystallization at $60^{\circ} \mathrm{C}$, respectively.

For the in-situ X-ray scattering measurements, the sensor holder with the FCC sensor plugged in is precisely positioned with the help of a special sample stage, including a hexapod and an array of piezo-actuators. The sample position can be monitored using an on-axis optical microscope, exchangeable with an X-ray detector. A single photon counting pixel array detector (Eiger $4 \mathrm{M}$ ) from Dectris was used for ensuring a combination of fast calorimetry with in situ X-ray scattering experiments using millisecond acquisition times. For both detectors several diffraction peaks of silver behenate and corundum were used to calibrate the norm of the scattering vector $\mathbf{q}(|\mathbf{q}|=4 \pi \sin (\theta) / \lambda$, where $\theta$ is the Bragg angle and $\lambda$ - the wavelength) in the small and wide angle regions. The acquired data was reduced and corrected using protocols provided by the beamline. The data analysis including two dimensional integration and visualization was done using home-built routines developed in the Igor Pro software package.

A typical P3EHT sample deposited on a MEMS-type FCC sensor is shown in Figure 1 (right). The size of the active area of the sensor is $100 \times 100 \mu \mathrm{m}^{2}$ and the sample mass is on the order of a few tens of nanograms. The synchronized fast heating experiments were realized at the rate of $100 \mathrm{~K} / \mathrm{s}$. The temperature scans at a conventional heating rate $(10 \mathrm{deg} / \mathrm{min})$ were performed at the BM26B beamlime of the ESRF using a commercial Linkam DSC stage.

\section{Results and discussion}

Typical 2D diffractograms measured on the micro-drops of P3EHT are shown in Figure 2 (left). The right panel of the same figure displays the corresponding 1D-resuced data measured upon crystallization at 25 and $60^{\circ} \mathrm{C}$. The indexation of the diffraction peaks has been performed according to the triclinic unit cell reported in ref. 3. The most intense diffraction peak of P3EHT is the lowangle 100 peak reflecting the stacking direction approximately along the alkyl side chains of the polymer. It can be seen that the unit cell of P3EHT does not change in the studied temperature range, however the diffraction peaks at $60{ }^{\circ} \mathrm{C}$ become sharper, the fact that reflects larger crystal size and higher crystal perfection. 

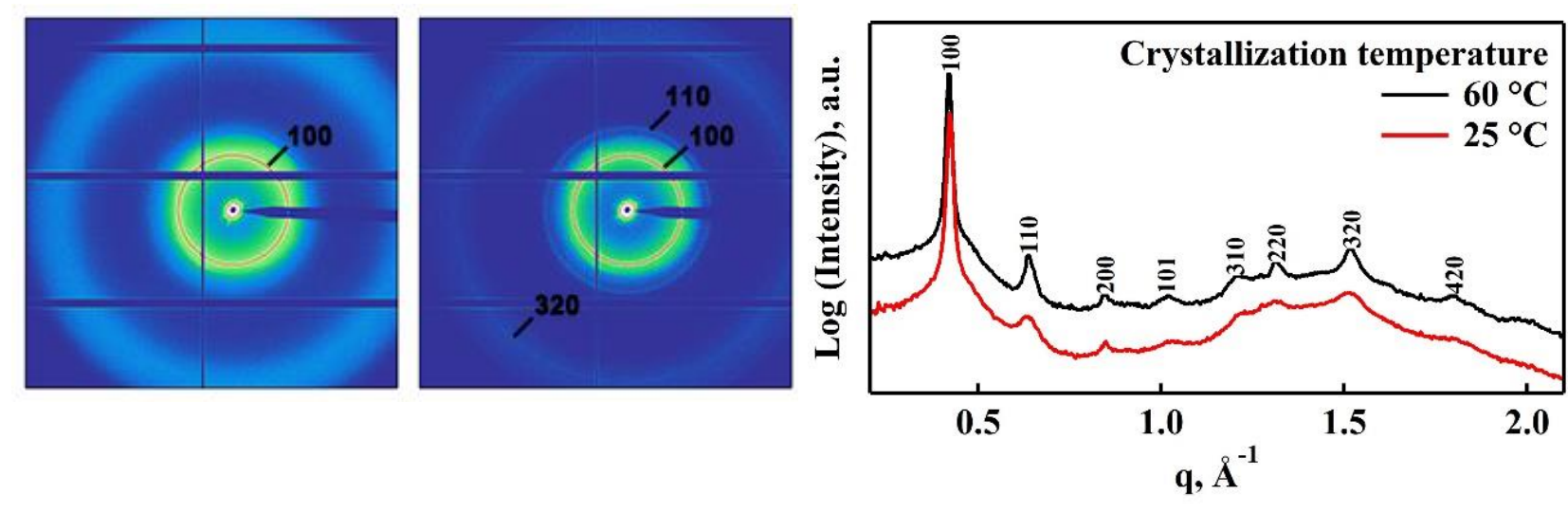

Figure 2. Left: 2D diffractograms measured on the P3EHT sample as shown in Fig. 1 at 25 and $60^{\circ} \mathrm{C}$. Right: 1D-reduced diffractograms calculated from the patterns in the left panel.

The kinetics of the melt crystallization can be addressed with FCC by measuring the melting enthalpy $\Delta \mathrm{H}_{\mathrm{m}}(\mathrm{t})$ developed after different crystallization times. The normalized degree of crystallinity $\alpha_{\mathrm{c}}(\mathrm{t})$ can be defined as:

$\alpha_{c}(t)=\frac{\Delta H_{m}(t)}{\Delta H_{m}(\infty)}$

where $\Delta \mathrm{H}_{\mathrm{m}}(\infty)$ denoted the melting enthalpy corresponding to very long crystallization time. Therefore, constructing the crystallization kinetics plot pertinent to each crystallization temperature requires performing several (ca. 10) measurements of the melting enthalpy by interrupting the process, cooling the sample to room temperature and heating it above the melting transition.

The resulting kinetics plots are shown in Figure 3 together with the fits to Avrami equation. The fitting equation reads as follows:

$\alpha_{c}(t)=1-\exp \left(-\left(\frac{t-t_{0}}{\tau}\right)^{n}\right)$

In eq.(2) $\tau$ stands for the characteristic crystallization time, $n$ is the Avrami exponent and $t_{0}$ is the induction time. It can be seen that in the temperature range from 25 to $65^{\circ} \mathrm{C}$ the rate of crystallization passes through a maximum. Tis can be accounted for by the fact that at the lower bound of crystallization temperatures they are approaching the glass transition at which all the motions in the polymer cease (kinetic limitation). At the higher bound of the temperature range, the crystallization kinetics is slowed down because the temperatures approach the final melting point (thermodynamic limitation). The temperature dependence of $\tau$ is shown in the inset of the figure. One can see that the values of $\tau$ pass through a minimum at approx. $55{ }^{\circ} \mathrm{C}$, which is roughly in the middle of the temperature range between the glass transition and melting. The values of the Avrami exponent are close to 1 . This likely indicates on 1D crystal growth geometry (formation of needle-like crystals). 


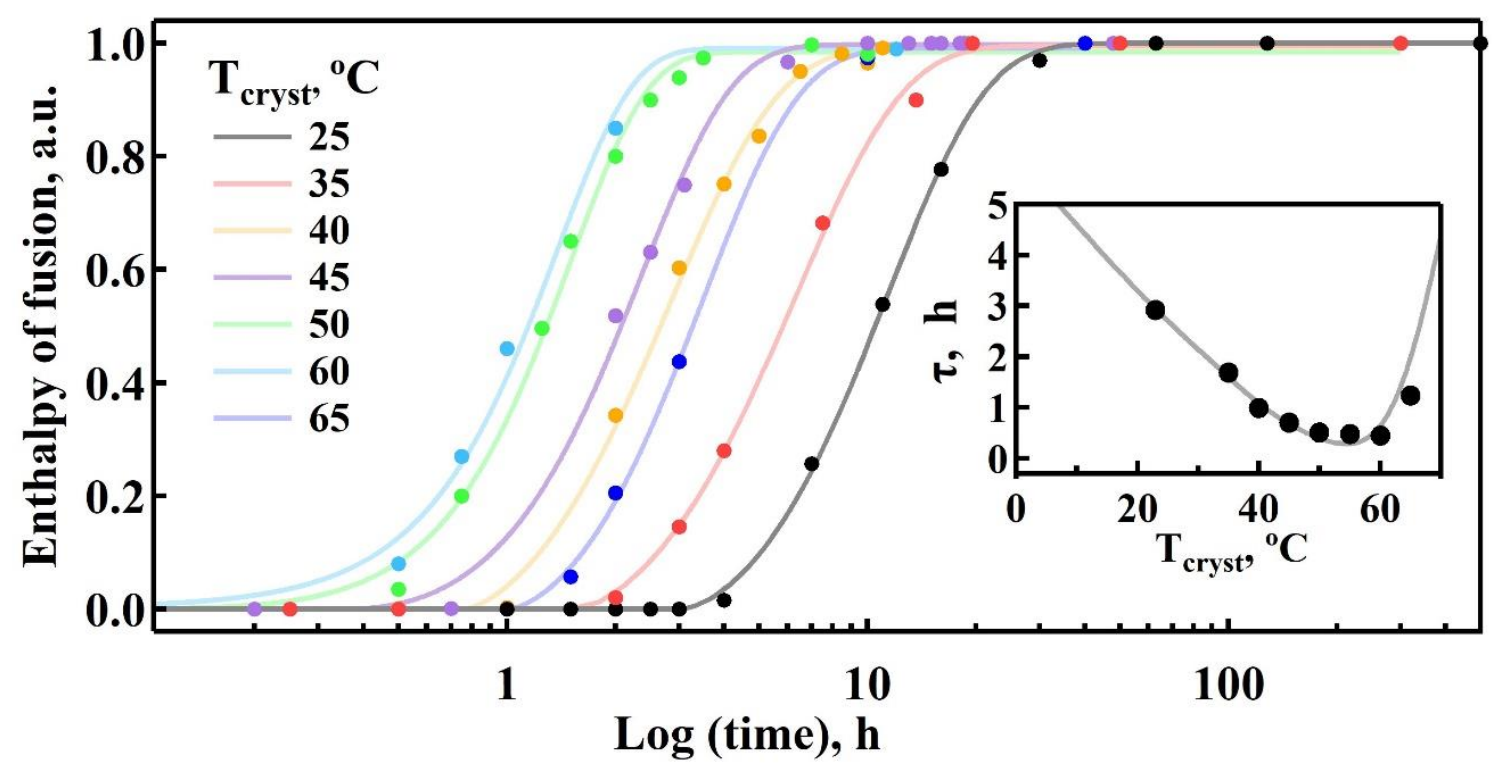

Figure 3. Kinetics of isothermal melt-crystallization of P3EHT measured with FCC. The inset displays the characteristic time of crystallization $\tau$ expressed in the units of seconds to the power of $1 / \mathrm{n}$, where $\mathrm{n}$ - is the Avrami exponent extracted from the fits to Avrami equation (solid lines).

The results of the combined X-ray scattering and calorimetry experiments are shown in Figure 4. The experiments have been performed on the P3EHT sample melt-crystallized for $24 \mathrm{~h}$ at $25^{\circ} \mathrm{C}$ and for 3 hours at $60{ }^{\circ} \mathrm{C}$. The conventional calorimetric experiments have been carried out at $10 \mathrm{deg} / \mathrm{min}$ whereas the fast scanning experiments has been conducted at $100 \mathrm{deg} / \mathrm{s}$ (or $6000 \mathrm{deg} / \mathrm{min}$ ) with the help of our home-built FCC accessory. The panels of Figure 4 display the intensity and spacing of the most intense 100 peak as well as the calorimetric signal.

It can be seen that the calorimetric curves (with the exception of the curve at $100 \mathrm{deg} / \mathrm{s}$ corresponding to the $25{ }^{\circ} \mathrm{C}$ crystallization) exhibit two and more melting peaks. Given that P3EHT does not display any polymorphic behaviour in the studied temperature range this puts this polymer among the ones clearly exhibiting the multiple-melting behavior. The low-temperature endothermic peak appears in the range of the crystallization temperature employed, whereas the high-temperature endothermic peak reflects the final melting of the polymer and can be located at a much higher temperature. Additional endothermic peaks can also appear in-between the two peaks. Thus, one can see that a third melting peak is visible for the case of crystallization at $25^{\circ} \mathrm{C}$ and low heating rate. Importantly, for the two cases studied, the final melting peaks recorded at the lowest heating rate are located higher than the ones recorded at the highest heating rate. This indicates on a likely reorganization/recrystallization of the sample at $10 \mathrm{deg} / \mathrm{min}$. The temperature difference between the peak positions is most pronounced for crystallization at $25{ }^{\circ} \mathrm{C}$. In this case, the sample can significantly reorganize on heating whereas for the other case the crystals grown at $60{ }^{\circ} \mathrm{C}$ are already close to their highest achievable thermodynamic stability.

The nature of the endothermic peaks can be addressed using the complementary X-ray scattering data measured simultaneously with the thermal signal. The intensity of the 100 peak reveals steps at the temperature positions of the endothermic peaks testifying that these peaks correspond indeed to melting and not to any kind of relaxation processes, as sometimes is assumed in the literature [14]. The evolution of the $\mathrm{d}_{100}$ spacing can provide additional information on the nature of the reorganization processes occurring in the course of heating. 
Indeed, it can be seen that for the low heating rate the slope of the $d_{100}$ spacing with temperature changes at the positions of the melting peaks. The increase of this distance was previously associated with melting of the alkyl side chains [15], which can in principle bring about an increase of the corresponding lattice parameter. However, the increase of the $\mathrm{d}_{100}$ spacing and its slope on the temperature plot can be also related to recrystallization and formation of new populations of crystals. Such recrystallization signature was previously identified by us for the case of poly(trimethylene terephthalate) (PTT) [10]. The experiments carried out at high heating rates do not reveal any stepwise increases of $\mathrm{d}_{100}$. This likely indicates that the critical heating rate at which the reorganization processes cease to exist is in-between the applied rates. The fact that the double-melting behavior can be observed at high heating rates above the critical one unambiguously show that the double melting behavior is not coupled to the reorganization process and can be considered as an intrinsic thermodynamic feature of this polymer family.

Interestingly, after initial increase of $\mathrm{d}_{100}$ probably related to a simple thermal expansion of crystals it decreases in the final melting range. Such final decrease may corroborate the model of the negative pressure exerted on the crystals in the later stages of crystallization, as proposed previously for PTT [16]. It might be that the final melting process releases the negative pressure imposed on the crystals by its neighbors and can result in the decrease of the corresponding spacing.

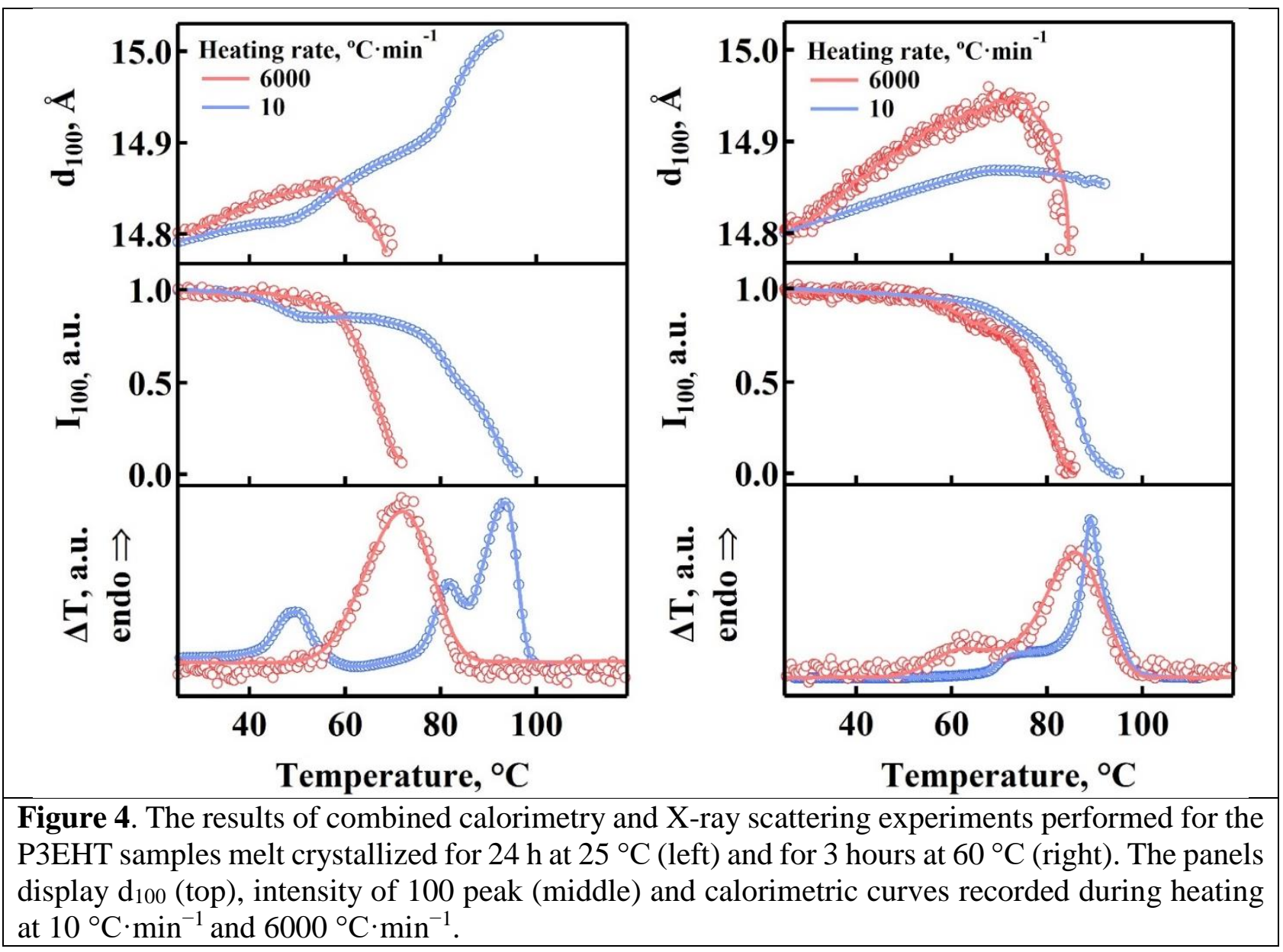

\section{Conclusions}

The thermal properties of an interesting analogue of the classical organic semiconductor P3HT poly(3-(2'-ethyl)hexylthiophene) were analysed using a combination of synchrotron X-ray scattering and ultrafast chip calorimetry. The signature of reorganization has been identified from an increase of d-spacing of 100 peak of the P3EHT unit cell. It was observed that reorganization operates during 
heating of P3EHT at conventional rates of a DSC experiment (i.e., at $10 \mathrm{deg} / \mathrm{min}$ ), whereas it is largely suppressed at a heating rate of $100 \mathrm{deg} / \mathrm{s}$. Despite the absence of reorganization at high heating rates the calorimetric curves exhibit pronounced double melting, which corroborates the model of the negative pressure building up during later-stage crystallization of semi-rigid chain polymers.

\section{Acknowledgments}

The authors greatly thank the Ministry of education and science of the Russian Federation (contract \#05.605.21.0188 from 3 December 2019 (RFMEFI60519X0188)) for financial support. The work was done on the theme of state task № 0074-2019-0014 (registration № AAAA-A19-1191015900290 ). The authors acknowledge excellent technical support provided by BM26B and ID13 beamlines stuff of ESRF (Grenoble). 


\section{References}

[1] B. C. Thompson, J. M. Frechet, Polymer-fullerene composite solar cells, J. Angew. Chem. Int. Ed. 47 (2008) 58-77.

[2] H. Sirringhaus, P. J. Brown, R. H. Friend, M. M. Nielsen, K. Bechgaard, B. M. W. LangeveldVoss, A. J. H. Spiering, R. A. J. Janssen, E. W. Meijer, P. Herwig, D. M. de Leeuw, Two-dimensional charge transport in self-organized, high-mobility conjugated polymers, Nature, 401 (1999) 685-688.

[3] B.W. Boudouris, V. Ho, L.H. Jimison, M.F. Toney, A. Salleo, R.A. Segalman, Real-Time Observation of Poly(3-alkylthiophene) Crystallization and Correlation with Transient Optoelectronic Properties, Macromolecules, 44 (2011) 6653-6658.

[4] B.S. Beckingham, V. Ho, R.A. Segalman, Melting Behavior of Poly(3-(2'ethyl)hexylthiophene). Macromolecules, 47 (2014) 8305-8310.

[5] M.Sh. Yagfarov, E.P. Mitrofanova, Recrystallization and secondary crystallization in polymers, Polymer Science USSR, 28(5) (1986) 1011-1017.

[6] Y. Lee, R.S. Porter, Double-melting behavior of poly(ether ether ketone), Macromolecules, 20 (1987) 1336-1341.

[7] D.A. Ivanov, A.M. Jonas, Isothermal growth and reorganization upon heating of a single poly(aryl-ether-ether-ketone) (PEEK) spherulite, as imaged by atomic force microscopy, Macromolecules 31 (1998) 4546-4550.

[8] D.A. Ivanov, A.M. Jonas, R. Legras, The crystallization of poly(aryl-ether-ether-ketone) (PEEK). Reorganization processes during gradual reheating of cold-crystallized samples, Polymer 41 (2000) 3719-3727.

[9] A.P. Melnikov, M. Rosenthal, A. Rodygin, D. Doblas, D. Anokhin, M. Burghammer, D.A. Ivanov, Re-exploring the double-melting behavior of semirigid-chain polymers with an in-situ combination of synchrotron nano-focus X-ray scattering and nanocalorimetry, Eur. Polym. J. 81 (2016) 598-606.

[10] A.P. Melnikov, M. Rosenthal, D.A. Ivanov, What Thermal Analysis Can Tell Us about Melting of Semicrystalline Polymers: Exploring the General Validity of the Technique, ACS Macro Lett. 7 (2018) 1426-1431.

[11] N. Piazzon, M. Rosenthal, A. Bondar, D. Spitzer, D.A. Ivanov, Characterization of explosives traces by the Nanocalorimetry. J. Phys. Chem. Solids 71 (2010) 114-118.

[12] M. Rosenthal, A.P. Melnikov, A.A. Rychkov, D. Doblas, D. Anokhin, M. Burghammer, D.A. Ivanov, Design of an In Situ Setup Combining Nanocalorimetry and Nano- or Micro-focus XRay Scattering to Address Fast Structure Formation Processes, Fast Scanning Calorimetry, Chapter: Chapter 9, Springer (2016).

[13] A.A. Rychkov, A.P. Melnikov, A.I. Rodygin, D.V. Anokhin, M. Rosenthal, D.A. Ivanov Ultra-fast chip calorimetry accessories for in operando structural studies of nanogram-sized samples, IOP Conf. Ser.: Mat. Sci. and Eng., 525 (2019).

[14] B.S. Beckingham, V. Ho, R.A. Segalman, Formation of a Rigid Amorphous Fraction in Poly(3-(2'ethyl)hexylthiophene), ACS Macro Lett., 3 (2014) 684-688.

[15] H. Chena, C. Sua, G. Shia, G. Liua, D. Wang, Nature of the double melting peaks of regioregular poly(3-dodecylthiophene), European Polymer Journal, 99 (2018) 284-288.

[16] D.A. Ivanov, G. Bar, M. Dosière, M.H.J Koch, A Novel View on Crystallization and Melting of Semirigid Chain Polymers: The Case of Poly(trimethylene terephthalate), Macromolecules, 41 (2008) 9224-9231. 